\title{
THEORETICAL AND EXPERIMENTAL STUDY OF A SHOCK TUBE PERFORMANCE WITH HOMOGENEOUS NUCLEATION OF WATER VAPOUR
}

\author{
N. H. MAHMOUD \\ Department of Mechanical Power Engineering, \\ Faculty of Engineering, Minufiya University, \\ Shebin El-Kom, EGYPT.
}

\begin{abstract}
:
Performance characteristics of a shock tube has been investigated here theoretically and experimentally when the homogeneous nucleation appears in the expansion fan within the driver section. Nucleation of water vapour is simulated using the nonisothermal formulation of the classical theory of nucleation. Shock tube performance is represented with the aid of an analytical model combines its pressure ratios. A modified shock tube equipped with a convergent section of different geometries and joined the driven section to the tail pipe is used in the present experimental program. Pressure traces ahead and behind the convergent section are measured using three pressure transducers. Both measurement and prediction of shock tuke flows show the dependence of the pressure ratio across the incident moving shock weve in the driven section on the tube overall pressure ratio. It is noted also from the present predictions the pronounced effect of the shock tube overall pressure ratio on the nucleation characteristics (i.e., amount of subcooling, supersaturation ratio, critical size of nucleated droplets and rate of nucleation) of the water vapour in the driver section. The validity of the suggested model is tested by comparing between the predicted and measured pressure ratios. Another comparison has been carried out between the calculated values of the nucleation rate in a shock tube and the published measured ones. The two comparisons indicate reasonable agreements.
\end{abstract}

KEYWORDS: Water vapour, condensation, shock tube, nucleation, shock wave, rarefaction wave, expansion fan.

Manuscript received from Dr. N.H. Mahmoud

Accepted on : $18 / 6 / 2001$

Engineering Research Journal Vol 24,No 3; 2001 Minufiya University, Faculty Of

Engineering, Shebien El-Kom, Egypt, ISSN 1110-1180 


\section{INTRODUCTION:}

When water vapour expands through nozzles or turbine blades, it supercools firstly, then nucleats and condenses lastly to form the wet steam mixture. Nucleation and condensation of water vapour are accompanied by thermal and inertial nonequilibriun effects, and erosion of nozzles or blades. Therefore, study of nucleation and condensation of water phase in wet steam is of particular importance in the design and operation of large steam turbines used in power plants.

The classical theory of homogeneous nucleation is based on predicting the nucleation rates of condensing vapours. Establishment of this theory in its classical formulation [1] or in its statistical mechanical treatment [2] is confirmed by nucleation rate measurement which is considered as the basis of condensation experiments. Measurements of nucleation rate have been carried out firstly using cloud chambers [3]. Generally speaking, cloud chamber experiments were directed to study the fog formation and to estimate the critical supersaturation ratio of the tested vapour. Afterwards, nozzle experiments have been widely used in obtaining data on one or more of the following; location of Wilson line, size and number of formed fog droplets, appearance of shocks and periodic phenomena through the condensing vapour [4]. Lastly, shock tubes have often been used to study vapours condensation owing to the best control on the experimental conditions of these devices. Wegener and Lundquist [5] are the first in using this tool in condensation researches. They utilized the cooling capabilities of the unsteady expansion fan inside the driver section of a shock tube to condense water vapour at temperatures below $150 \mathrm{~K}$.

A shock tube consists mainly as shown diagrammatically in Fig. (1) of two sections of pipe; namely, the driver and the driven sections, separated by a diaphragm. The driver and driven sections are filled with the same gas (or vapour) with the pressure in the driver section higher than that in the driven section. Different discontinuities are appeared after the diaphragm has suddenly been removed. A shock wave moves to the right direction through the driven section. The shock wave intensity depends on the initial motion of the vapour inside the driver section, the pressure difference between the two sections as well as the temperature difference and the thermodynamic properties of the two masses of vapour before the diaphragm rupture [6]. When the shock wave crosses from the high pressure vapour into the low pressure one, an expansion wave travels to the left at a front speed $a_{4}$ followed by an expansion fan as shown by a fan of straight lines in Fig. (1). Many researches were directed to follow the pioneering work of Wegener and Lundquist [5] to utilize the cooling capabilities of the expansion fan between regions 4 and 3 \{Fig.(1)\} in understanding the condensation dynamics in the rarefaction situations. The first support to Wegener and Lundquist findings of water vapour condensation in a shock tube was presented by the first streak photographs of Glass and Patterson [7]. These photographs showed the condensation zones in the expansion fan.

Shock tube experiments with nonequilibrium condensation occurs in the rarefaction waves have been carried out for pure water vapour by many investigators (e.g., Barschdorff [8] and Sislian [9]). These experiments were directed to examine the condensation characteristics for small mole fractions of water vapour in different carrier gases $\{$ e.g., Wegener and Lee[10] and Peters [11]\}. It has been realized that very large 
values of overall pressure ratio $\left(P_{4} / P_{1}\right)$ are required to generate strong shock waves in such a shock tube. One way to improve the performance of the shock tube is to select a driver gas with a large speed of sound. Therefore, shock tube experiments are extended to study the homogeneous nucleation of xenon [12] and ethanol and n-propanol [13] as well as the effect of cooling rate on binary nucleation [14]. Finally, multiple-diaphragm versions of a large scale ( $0.5 \mathrm{~m}$ inner diameter and $200 \mathrm{~m}$ long) shock tube is used to study the time of existence of high-temperature working flows, contact zones and regions of uniform cold flow [15].

Modifications of the shock tube experiment has been of particular interest recently. In one of these modifications, nozzle flow is combined to the supersonic shock tube flow in order to obtain the so-called Ludwieg tube. This tube was designed and utilized firstly by Ludwieg as a special type of a hypersonic wind tunnel [16]. This device is based on the fact that if the shock wave propagates through a variable area duct, the strength of the shock wave will be changed. A detailed analysis of the interaction between a moving shock wave and the curved wall of a convergent nozzle located beyond the driven section of a shock tube, that operates with air was carried out by Nasr [17]. The experiments of Matthew and Steinwandel [18] and Steinwandel and Buchholz [16] show that introducing the nozzle section into Ludwieg tube seems to cause a substantial difference in the observed onset of condensation.

In this work, an analytical model is developed to simulate the relation between a shock tube pressure ratios and the nucleation of water vapour in the driver section. Besides, a shock tube equipped with a convergent section and a tail pipe is designed, constructed and utilized to obtain measured values for the pressure variation of the shock tube flows. The capability of the present model to simulate the shock tube performance is tested through comparisons between its predictions and the present experimental results as well as the published measurements.

\section{THEORETICAL MODEL :}

In the following a suggested analytical model simulates the main characteristics of the performance of such a shock tube is presented. This model is divided into two sections, the first one concerns with combining the pressure ratios across the tube sections while the second one deals with predicting the vapour nucleation inside the driver section. First of all, the assumptions which are applied to simplify the present model are listed below:

a- Shock tube flow may be considered unsteady, one-dimensional, inviscid and adiabatic.

b- Nucleation may occur in the driver section in the form of spherical droplets of the critical size corresponding to the vapour supersaturation ratio.

c- The nucleated droplets still in thermodynamic equilibrium with the supersaturated vapour (i.e., without condensation).

\subsection{Shock tube pressure ratios:}

Referring to Fig. (2), regions 1 and 4 of the shock tube are initially separated into high and low pressure sections by the diaphragm. At $t=0$ the diaphragm bursts and 
the high pressure driver gas rushes into the low pressure section. The contact surface between the two masses of gas acts like a piston. The conditions at the contact surface are

$$
\begin{aligned}
& P_{2}=P_{3} \\
& C_{2}=C_{3}
\end{aligned}
$$

In a frame of reference moving with the shock, the velocity behind the shock is given by

$$
C_{1}^{\prime}-C_{2}^{\prime}=C_{2}=a_{1}\left(\frac{P_{2}}{P_{1}}-1\right)\left[\frac{2 \gamma_{1}}{\left(\gamma_{1}+1\right) \cdot\left(\frac{P_{2}}{P_{1}}\right)+\left(\gamma_{1}-1\right)}\right]^{\frac{1}{2}}
$$

The velocity behind the expansion is expressed as

$$
C_{3}=\frac{2 a_{4}}{\gamma_{4}-1}\left[1-\left(\frac{P_{3}}{P_{4}}\right)^{\frac{\gamma_{4}-1}{2 \gamma_{4}}}\right]
$$

Now, equating $C_{3}$ and $C_{2}$ and solve for $P_{4} / P_{1}$ yields to

$$
a_{1}\left(\frac{P_{2}}{P_{1}}-1\right)\left[\frac{2 \gamma_{1}}{\left(\gamma_{1}+1\right)\left(\frac{P_{2}}{P_{1}}\right)+\left(\gamma_{1}-1\right)}\right]^{\frac{1}{2}}=\frac{2 a_{4}}{\gamma_{4}-1}\left\{1-\left[\left(\frac{P_{3}}{P_{2}}\right)\left(\frac{P_{2}}{P_{1}}\right)\left(\frac{P_{1}}{P_{4}}\right)\right]^{\frac{\gamma_{4}-1}{2 \gamma_{4}}}\right\}
$$

and

$$
a_{1}\left(\frac{P_{2}}{P_{1}}-1\left[\frac{2 \gamma_{1}}{\left(\gamma_{1}+1\right)\left(\frac{P_{2}}{P_{1}}\right)+\left(\gamma_{1}-1\right)}\right]^{\frac{1}{2}}=\frac{2 a_{4}}{\gamma_{4}-1}\left\{1-\left[(1)\left(\frac{P_{2}}{P_{1}}\right)\left(\frac{P_{1}}{P_{4}}\right)\right]^{\frac{\gamma_{4}-1}{2 \gamma_{4}}}\right\}\right.
$$

Rearranging Equ. (5) gives the basic shock tube equation in the form

$$
\frac{P_{4}}{P_{1}}=\frac{P_{2}}{P_{1}}\left\{1-\frac{\left(\gamma_{4}-1\right)\left(\frac{a_{1}}{a_{4}}\right)\left(\frac{P_{2}}{P_{1}}-1\right)}{\sqrt{2 \gamma_{1}} \sqrt{2 \gamma_{1}+\left(\gamma_{1}+1\right)\left(\frac{P_{2}}{P_{1}}-1\right)}}\right\}^{-\frac{2 \gamma_{4}}{\gamma_{4}-1}}
$$


Equation (6) was simplified by Wegener and Sreenivasan [14] on the basis of the assumption that the isentropic exponent of water vapour expansion is kept constant during the operation of the shock tube to obtain

$$
\frac{\mathbf{P}_{4}}{\mathbf{P}_{1}}=\frac{\mathbf{P}_{2}}{\mathbf{P}_{1}}\left\{1-\frac{(\gamma-1)\left(\frac{\mathbf{P}_{2}}{\mathbf{P}_{1}}\right)}{\sqrt{2 \gamma} \sqrt{2 \gamma+(\gamma+1)\left(\frac{\mathbf{P}_{2}}{\mathbf{P}_{1}}-1\right)}}\right\}^{-\frac{2 \gamma}{\gamma-1}}
$$

Equations (6) and (7) were estimated and utilized by many investigators \{ e.g., Barschdorff [8], Peters [11] and Wegener and Sreenivasan [14]\}. Considering the tail pressure $P_{3}$ to be still unchanged after the expansion, the tail temperature $T_{3}$ can be estimated using Poisson's law for an adiabatic expansion as

$$
\frac{T_{3}}{T_{4}}=\left[\frac{P_{3}}{P_{4}}\right]^{\frac{\gamma_{4}-1}{\gamma_{4}}}=\left[\frac{P_{3} / P_{1}}{P_{4} / P_{1}}\right]^{\frac{\gamma_{4}-1}{\gamma_{4}}}=\left[\frac{P_{2} / P_{1}}{P_{4} / P_{1}}\right]^{\frac{\gamma_{4}-1}{\gamma_{4}}}
$$

Following the assumption of Wegener and Sreenivasan [14] (i.e., $\gamma_{1}=\gamma_{4}=\gamma$ and $a_{1}=a_{4}$ ), Equ. (8) becomes

$$
\frac{T_{3}}{T_{4}}=\left[\frac{P_{2} / P_{1}}{P_{4} / P_{1}}\right]^{\frac{\gamma-1}{\gamma}}
$$

During computations with Equ. (6), the frozen speeds of sound $a_{1}$ and $a_{4}$ have been obtained in both the equilibrium and nonequilibrium wet steam situations by

$$
\begin{aligned}
& a_{1}=\sqrt{\gamma_{1} \cdot R_{g} \cdot T_{1}} \\
& a_{4}=\sqrt{\gamma_{4} \cdot R_{g} \cdot T_{4}}
\end{aligned}
$$

\subsection{Nucleation characteristics of the driver section:}

After diaphragm rupture; an unsteady, isentropic expansion propagates through the driver section. During this expansion, vapour pressure inside the driver decreases monotonically and the vapour becomes supersaturated. Upon arrival of the head of the expansion fan to a such location $\left(x_{o b s}\right)$ inside the driver \{see Fig. (1)\}, nucleation will appear as detected by light scattering[8,10\&11]. At this location, a fine fog consisting of tiny droplets is being formed in this region across the entire driver cross section. Nucleation rate in this fog is defined as the number of supercritical-sized droplets 
generated per unit mass (or unit volume) per unit time. Based on the assumptions of the classical theory of nucleation [4], nucleation rate takes the form

$$
I=\sqrt{\frac{2 \sigma}{\pi m_{m}^{3}}} \cdot \frac{\rho_{g}}{\rho_{f}} \exp \left(-\frac{4 \pi r_{*}^{2} \sigma}{3 k T_{g}}\right)
$$

or

$$
J=\sqrt{\frac{2 \sigma}{\pi m_{m}^{3}}} \cdot \frac{\rho_{g}^{2}}{\rho_{f}} \exp \left(-\frac{4 \pi r_{*}^{2} \sigma}{3 k T_{g}}\right)
$$

where $\mathrm{I}$ and $\mathrm{J}$ are the nucleation rate in $\mathrm{kg}^{-1} \cdot \mathrm{s}^{-1}$ and $\mathrm{m}^{-3} \cdot \mathrm{s}^{-1}, \sigma$ is the surface tension on the droplet surface, $m_{m}$ is the mass of one molecule and $\mathrm{k}$ is the Boltzmann's constant.

The present model uses this classical formulation for the nucleation rate together with the last formulation of this rate which is denoted as "the nonisothermal nucleation rate" and presented by Young [19] as

$$
I_{n i}=\frac{1}{1+\phi} \cdot I
$$

or

$$
J_{n i}=\frac{1}{1+\phi} . J
$$

where I and J are calculated from Equs. (12) and (13) and the coefficient $\phi$ takes the following definition

$$
\phi=2\left(\frac{\gamma-1}{\gamma+1}\right) \cdot\left(\frac{h_{f g}}{R_{g} \cdot T_{g}}\right) \cdot\left(\frac{h_{f g}}{R_{g} \cdot T_{g}}-\frac{1}{2}\right)
$$

The critical size of the nucleated droplets is given for ideal-gas vapours by

$$
r_{*}=\frac{2 \sigma}{\rho_{f} \cdot R_{g} \cdot T_{g} \cdot \ln S}
$$

The supersaturation ratio in Equ. (17) is defined as

$$
S=\frac{P}{P\left(T_{g}\right)}
$$


When the combination between the supersaturation ratio and the amount of subcooling is required during the calculations, the following relation which was obtained by Gyarmathy [4] is used in the present model

$$
\log S=-\frac{2061}{T_{s}}+\frac{2061}{T_{g}}=\frac{2061}{T_{s} T_{g}} \cdot \Delta T
$$

Finally, the accumulated mass of the nucleated droplets within the supersaturated vapour of the driver section is represented by the so-called "wetness fraction" which takes the form

$$
Y=\frac{4 \pi}{3} \rho_{f} \cdot r_{*}^{3} \cdot I . \tau
$$

where $\tau$ is the time of expansion fan propagation through the driver section and defined by

$$
\tau=L_{D} / C_{3}
$$

In Equ. (21), $L_{D}$ is the length of the driver section and $C_{3}$ is the velocity of the expansion wave. Based on the present assumptions and using isentropic flow relations for a rarefaction wave, the following relation is used to compute $C_{3}$

$$
\frac{C_{3}}{a_{4}}=\frac{2}{\gamma_{4}-1}\left(1-\frac{a_{3}}{a_{4}}\right)
$$

The frozen speed of sound $a_{3}$ in Equ. (22) is given under the assumption of constant isentropic index of wet steam expansion by

$$
a_{3}=\sqrt{\gamma \cdot R_{g} \cdot T_{3}}
$$

In order to solve the above system of equations numerically, both the isentropic exponent of wet steam expansion and water surface tension are required. The isentropic exponent $\gamma$ is taken either as a constant value or a variable value. The constant value of $\gamma$ taken is 1.135 due to Gyarmathy [4] or 1.32 as suggested by Yeoh and Young [20]. The variable values of $\gamma$ are computed using the following formula which was presented by Nicola [21]

$$
\gamma=0.603+a_{o}\left[1-\left(\frac{P}{P_{*}}\right)^{3 / 2}\right]^{a_{e}}
$$

where $\cdot a_{o}=0.522-0.1418 Y /(1-Y)$ 


$$
, \quad a_{e}=1.34565-0.76825(1-Y)
$$

Whilst the surface tension on the water droplet surface is calculated from a formula published by Tolman [22] to combine between the surface tension on the droplet surface and the bulk surface tension (i.e., the surface tension on a flat water surface) namely

$$
\frac{\sigma}{\sigma_{\infty}}=\frac{1}{1+\frac{2 \delta}{r}}
$$

where $\delta$ is a constant corresponding to the condensed vapour and $\sigma_{\infty}$ is the bulk surface tension which expressed by Jaeger et al [23] for water as

$$
\sigma_{\infty}=\sigma_{a}-\sigma_{b}(T-273)
$$

where $\sigma_{a}=75.7 \mathrm{dyn} / \mathrm{cm}$ and $\sigma_{b}=0.177 \mathrm{dyn} / \mathrm{cm} . \mathrm{K}$.

The estimated values of $\sigma_{\infty}$ from Equ. (26) are in dyn $/ \mathrm{cm}$ when $\mathrm{T}$ is in K. A similar formula was presented by Lee and Tankin [24].

Computation of $\left(P_{2} / P_{1}\right)$ is carried out for the applied initial pressure ratio $\left(P_{4} / P_{1}\right)$ by solving Equ. (6) or Equ. (7) through iterative procedure. During the iterations, the frozen speeds of sound $a_{1}$ and $a_{4}$ as well as the isentropic indecies of wet steam expansion $\gamma_{1}$ and $\gamma_{4}$ are calculated according to the applied assumptions with the aid of Equs. (10), (11) and (24). Nucleation characteristics of the steam in the driver section are being computed for the tested boundary conditions through solving Equs. (12-20) in conjunction with the supplementary Equs. (21-26). The temperature $T_{g}$ in Equs. (10-13) and (16-19) is taken here equals $T_{3}$ and obtained from Equs. (8) or (9).

\section{EXPERIMENTAL APPARATUS AND MEASUREMENTS:}

In Fig. (3), a general layout is presented for the experimental set-up of the present work. The set-up compromises a fire-tube boiler of 1 ton/hr capacity supplies a shock tube with wet steam of 0.995 dryness fraction at 6.0 bar. The shock tube consists of three sections; the first one involves the driver and driven sections, the second one is a convergent section and the third one is a tail pipe. The driver and driven sections were made of two tubes of $1.6 \mathrm{~m}$ length and $82.5 \mathrm{~mm}$ inner diameter for each and separated by a diaphragm. The tail pipe was made of a pipe of $1.5 \mathrm{~m}$ length and $50.8 \mathrm{~mm}$ inner diameter. The driver, driven and tail pipes were made of steel 37 . The diaphragm separating the driver and driven sections was ruptured using a needle connected to an external mechanism.

Prior to a test run, the shock tube was heated by steam until its pressure reaches the value of 1.0 bar. After closing the supply and drain valves, the steam pressure inside the driver section was raised to the tested initial value $\left(P_{4}\right)$ whilst the steam pressure in the driven section $\left(P_{1}\right)$ was kept constant at 1.0 bar. The pressure $P_{1}$ in the driven section was adjusted using steam feedback through the tail pipe as shown in Fig. (3). 
Upon breaking the diaphragm, the shock wave propagation inside the driven section is caught with the aid of pressure measurement instrumentation.

Three pressure transducers and a storage oscilloscope were used to record the pressure-time history of both the incident shock wave ahead the convergent section and the transmitted shock wave inside the tail pipe. Pressure taps of inner diameter equals $10.0 \mathrm{~mm}$ were drilled along the driven section and the tail pipe in order to change the locations of the pressure transducers. Pressure transducers used here are of model SA PSIS/PSIA from Data Instruments, Lexington, USA. The range of transducers measurement is of $0: 150$ psia and with accuracy of $\pm 0.5 \%$ of full scale. One of these transducers was used as a triggering device and the others were used to measure the static pressure in two locations upstream and downstream the convergent section. The output signals from the transducers were displayed on a four channel oscilloscope ( type Tektronix 15223 digitizing oscilloscope). The displayed signals were stored and photographed in each run. Steam pressure inside the driver section was measured using a Bourdon-tube pressure gauge of readability from 0 to 6 bar and scale intervals equals 0.1 bar. Finally, steam dryness fraction was evaluated at the shock tube entrance using a throttling calorimeter.

The ambient temperature and atmospheric pressure were uniform within $35 \pm 1 C^{\circ}$ and $750 \pm 2 \mathrm{~mm} \mathrm{Hg}$. The uncertainty in the measured values of $P_{4}$ was within a maximum value of $\pm 2.5 \%$ and a minimum value of $\pm 1.0 \%$. Whilst the uncertainty in the pressure measurement using the pressure transducers was in the range of $\pm 0.5 \%$ of full scale. Furthermore, the uncertainty in measuring steam dryness fraction was obtained to be about $\pm .0 .05 \%$.

\section{RESULTS AND DISCUSSION:}

The theoretical results plotted in Figs. (4-7) were obtained for the operation of a basic shock tube which is illustrated in Fig. (2). It is of great importance to mention here that all the predictions in Figs. (4-7) were obtained under the assumption that the steam filled the shock tube sections before operation (i.e., at $\mathrm{t}=0$ ) was dry and saturated steam.

Figure (4) presents the dependence of the pressure ratio across the incident shock wave or the so-called the shock strength $\left(P_{2} / P_{1}\right)$ on the initial pressure ratio of the shock tube $\left(P_{4} / P_{1}\right)$ for different values of the isentropic exponent of wet steam expansion. It is apparent from this figure that $\left(P_{2} / P_{1}\right)$ increases monotonically with the increase of $\left(P_{4} / P_{1}\right)$. However, changing the isentropic exponent $(\gamma)$ from dependent pressure values to constant ones of 1.135 and 1.32 results in small differences at initial pressures less than 10.0 bar. These differences between curves in Fig. (4) tends to increase with increasing the initial pressure $P_{4}$ from 10.0 to 80.0 bar. Plots of Fig. (4) show also that single phase medium $(\gamma=1.4)$ requires higher values of the initial pressure to perform the same shock strength when compared with wet steam medium of lower $\gamma$ values. Owing to the importance of the pressure and temperature at the expansion wave tail (i.e., $P_{3}$ and $T_{3}$ ), the predicted values of $P_{3}$ and $T_{3}$ as well as $P_{2} / P_{1}$ are shown with the changes of $P_{4} / P_{1}$ in Fig. (5). Predictions of Fig. (5) were obtained from solving Equ. (7) at a constant value of $\gamma$ equals 1.135. It is noted from 
the figure that $P_{2} / P_{1}, P_{3}$ and $T_{3}$ are increased with increasing $P_{4} / P_{1}$. It is worth mentioning here that this dependence of $P_{3}$ and $T_{3}$ on the initial pressure ratio was discussed previously by Peters [11].

Effect of the initial pressure ratio on the condensation characteristics of the steam inside the driver section of a basic shock tube is given in Fig. (6). It is obvious in this figure that as $P_{4} / P_{1}$ increases the predicted critical size of the nucleated droplets decreases and then increases while the amount of subcooling, supersaturation ratio and nucleation rate are increased. The reason for these tendencies is explained by the dependence of $T_{3}$ on $P_{4} / P_{1}$. As was concluded from the discussion of Fig. (5) about $T_{3}$ ( i.e., $T_{3}$ increases with increasing $P_{4} / P_{1}$ ), it should be noted that as $P_{4}$ increases the predicted vapour temperature $T_{3}$ deviates more from the saturation temperature corresponding to $P_{2}$ (with remembering that $P_{2}=P_{3}$ ). Therefore, the computed critical radius of the nucleated droplets from Equ. (17) decreases with increasing $T_{g}$ (i.e., $T_{3}$ ). Thus increasing $P_{4} / P_{1}$ causes to increase $P_{3}$ as shown in the discussion of Fig. (5). Consequently, both $\mathrm{S}$ and $\Delta T$ are increased. The increase of $r_{*}$ in the range $5.0>\left(P_{4} / P_{1}\right)<6.0$ can be attributed to the change in the rate of $\Delta T$ and $\mathrm{S}$ increasing. For $\left(P_{4} / P_{1}\right)$ values less than 5.0, it can be noticed in Fig. (6.a) that the rate of $\Delta T$ and $\mathrm{S}$ increasing has higher values comparing to the feeble ones at $\left(P_{4} / P_{1}\right)>5.0$.

In Fig. (7) variations of the predicted condensation characteristics with different steam pressures in the driver section of a basic shock tube are presented. Theoretical results plotted in Fig. (7) indicate that, as the steam supersaturation ratio increases both the vapour pressure and critical radius of nucleated droplets are decreased when the driver pressure is kept constant. Besides, as S increases in Fig. (7.b) the nucleation rate and wetness fraction are increased. Behaviours of $P\left(T_{g}\right), r_{*}, I$ and $\mathrm{Y}$ in Fig. (7) coincide with the basic rules of wet vapours condensation that include "higher nucleation rates and wetness fractions as well as lower vapour pressures and critical droplet sizes can be achieved in the situations of higher degrees of supersaturation" [4].

Measurements in Fig. (8) illustrate the influence of both the convergent section geometry and the initial pressure ratio on the strengths of the incident and transmitted shock waves inside the tested shock tube. Plots of Fig. (8) show the increase of $P_{2} / P_{1}$ and $P_{T} / P_{1}$ with the increase of $P_{4} / P_{1}$. This dependence was observed previously in the present predictions $\left\{\right.$ i.e., increasing $P_{2} / P_{1}$ when $P_{4} / P_{1}$ was increased in Figs. (4-5)\}. It can be noticed in Fig. (8.a) also that the convergent section geometry did not affect the strength of the incident shock wave inside the driven section. But for the measured strengths or pressure ratios $\left(P_{T} / P_{1}\right)$ across the transmitted shock waves within the tail pipe, it is apparent the deviations between the measured values of $P_{r} / P_{1}$. This is because the rate of area change $(\mathrm{dA} / \mathrm{dL})$ is higher in the case of concave and convex geometries than that of the conical geometry.

Finally, comparisons between the suggested model predictions and both the present measurements and the published experimental results are carried out here to check the model validity. These comparisons are presented in Fig. (9). Figure (9.a) 
illustrates a comparison between the model predictions for the strength of the incident shock wave $\left(P_{2} / P_{1}\right)$ and the present measurements. Comparison of Fig. (9.a) reveals reasonable agreement. Deviations between the predicted and measured strengths of the incident shock waves may attributed to the accuracy of the utilized pressure transducers in detecting the pressure across the shock. Another comparison was carried out between the measurements of Peters [11] for both the nucleation rate and the corresponding supersaturation ratio and the present computations. This comparison is illustrated in Fig. (9). This comparison indicates a quite good agreement between measurements and present predictions. This means that the assumption of using the nonisothermal formulation of the nucleation rate improves the prediction of vapours nucleation inside shock tubes.

\section{CONCLUSION:}

The results of the present research indicate that shock tube can be used to facilitate the investigation of the problem of vapour condensation. The aerodynamic characteristics of a system of waves including expansion, incident, reflected and transmitted ones can be studied using shock tube. The present model predictions correlate the pressure ratios of such a shock tube. It can also be concluded that increasing the shock tube initial pressure ratio causes an increase in the strength of the incident moving shock wave inside the driven section of the tube. This finding is confirmed experimentally by the present measurements. Furthermore, the present measurements reveal the same dependence of the strength of the transmitted shock wave within the tail pipe on the initial pressure ratio. It is of great importance to conclude also that steam nucleation in rapid expansions at low initial pressures can be well predicted for technological applications using the present model.

\section{NOMENCLATURE:}

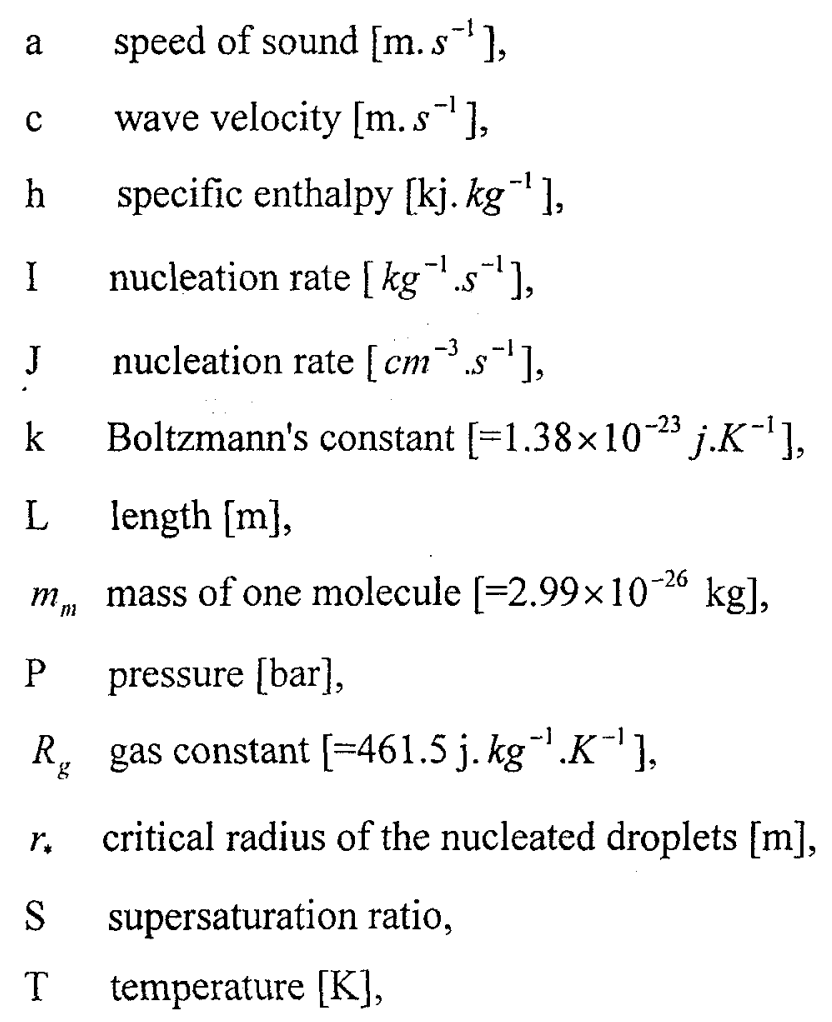


$\mathrm{Y}$ wetness fraction,

$\gamma \quad$ index of isentropic expansion,

$\rho$ density $\left[\mathrm{kg} \cdot \mathrm{m}^{-3}\right]$,

$\sigma \quad$ surface tension [dyn. $\mathrm{cm}^{-1}$ ],

$\tau$ time in Equ (21) [s],

$\phi$ dimensionless coefficient defined by Equ. (16).

\section{Subscripts}

1 driven section of the shock tube, initial state

2 state behind the incident shock wave

3 state at tail of the expansion wave

4 driver section of the shock tube, initial state

D driver

f water

fg phase transition

g vapour, gas

ni nonisothermal

s saturation

T transmitted

$\infty$ flat surface

\section{REFERENCES:}

1. Frenkel, J., "Kinetic theory of liquids", Oxford University Press, Dover Reprint, 1955.

2. Lothe, J. and Pound, G. M., "Statistical mechanics of nucleation" in: "Nucleation", Edited by: Zettlemoyer, A. C., Marcell Dekker, New York, 1969.

3. Andres, R. P., "Homogeneous nucleation from the vapour", in : "Nucleation", Edited by: Zettlemoyer, A. C., Marcell Dekker, New York, 1969.

4. Moore, M. J. and Sieverding, C. H. (Eds.), "Two-phase steam flow in turbines and separators", Hemisphere Publ., Washington, 1976.

5. Wegener, P. and Lundquist, G. ,"Condensation of water vapour in the shock tube below 150 K", J. Appl. Phys., Vol. 22, p. 233, 1951.

6. Sedov, L. I., "Similarity and dimensional methods in mechanics", Mir Publ., Moscow, 1982.

7. Glass, I. I. and Patterson, G. N., "A theoretical and experimental study of shock tube flows", J. Aero. Sci., Vol. 22, pp. 75-100, 1955. 
8. Barschdorff, D., "Carrier gas effects on homogeneous nucleation of water vapour in a shock tube", Physics Fluids, Vol. 18, No. 5, pp. 529-535, 1975.

9. Sislian, J. P., "Condensation of water vapour with or without a carrier gas in a shock tube",Rep. 201, University of Toronto, Institute for Aerospace Studies, 1975.

10. Wegener, P. P. and Lee, C. F., "Condensation by homogeneous nucleation of $\mathrm{H}_{2} \mathrm{O}, \mathrm{C}_{6} \mathrm{H}_{6}, \mathrm{CCl}_{4}$ and $\mathrm{CCl}_{3} \mathrm{~F}$ in a shock tube", J. Aerosol Sci., Vol. 14, No. 1, pp. 29-37, 1983.

11. Peters, F. ,A new method to measure homogeneous nucleation rates in shock tubes", Experiments in Fluids, Vol. 1, pp. 143-148, 1983.

12. Zahoransky, R. A., "Homogeneous nucleation of xenon in supersonic shock tube flow", J. Chem. Phys., Vol. 82, No. 6, pp. 2783-2784, 1985.

13. Peters, F. "Homogeneous nucleation of ethanol and n-propanol in a shock tube", J. Chem. Phys., Vol. 77, No. 9, pp. 4788-4790, 1982.

14. Wegener, P. P. and Sreenivasan, K. R. ,"The effect of cooling rate on binary nucleation", Appl. Sci. Res., Vol. 37, pp. 183-194, 1981.

15. Anfimov, N. A., Ershov, I. V., Ruzavin, E. I., Semenov, S. S. and Shimarev, S. K., "Structure of hypersonic flows in a large-scale multiple diaphragm shock tube", Rossijskaya Akademiya Nauk, Mekhanika Zhidkostii Gaza (in Russian), No. 5, pp. 158-165, 1993.

16. Steinwandel, J. and Buchholz, T., "Homogeneous condensation of Argon : an experimental study using the nozzle flow of a cryogenic Ludwieg tube", Aerosol Sci. and Tech., Vol. 3, pp. 71-76, 1984.

17. Nasr, M., "An investigation of a shock wave propagation in a mildly and strongly curved wall convergent ducts", Eng. Res. Bull., Menoufia Univ., Vol. 18, Part 2, pp. 237-250, 1995.

18. Matthew, M. W. and Steinwandel, J., "An experimental study of argon condensation in cryogenic shock tubes", J. Aerosol Sci., Vol. 14, No. 6, pp. 755-763, 1983.

19. Young, J. B., "Wet steam flows", Cambridge University Lecture Series on Turbomachinery, Cambridge, UK, 1994.

20. Yeoh, C. C. and Young, J. B., "The effect of droplet size on the flow in the last stage of a one-third scale model low-pressure turbine", Proc. Instn Mech. Engrs, Vol. 198A, No. 13, pp. 309-316, 1984.

21. Nicola, M. C., "Der exponent der isentropishen expansion fur nassdampf und sattdampf", Warme, Band 71, Heft 2, 1971.

22. Tolman, R. C., "The effect of droplet size on surface tension", J. Chem. Phys., Vol. 17 , No. 3, pp. 333-337, 1949.

23. Jaeger, H. L., Willson, E. J. and Hill, P. G., "Nucleation of supersaturated vapours in nozzles. I. $\mathrm{H}_{2} \mathrm{O}$ and $\mathrm{NH}_{3}$ ", J. Chem. Phys., Vol. 51, No. 12, pp. 5380-5388, 1969.

24. Lee, S. Y. and Tankin, R. S., "Study of liquid spray (water) in a condensable environment (steam)", Int. J. Heat Mass Transfer, Vol. 27, No. 3, pp. 363-374, 1984. 


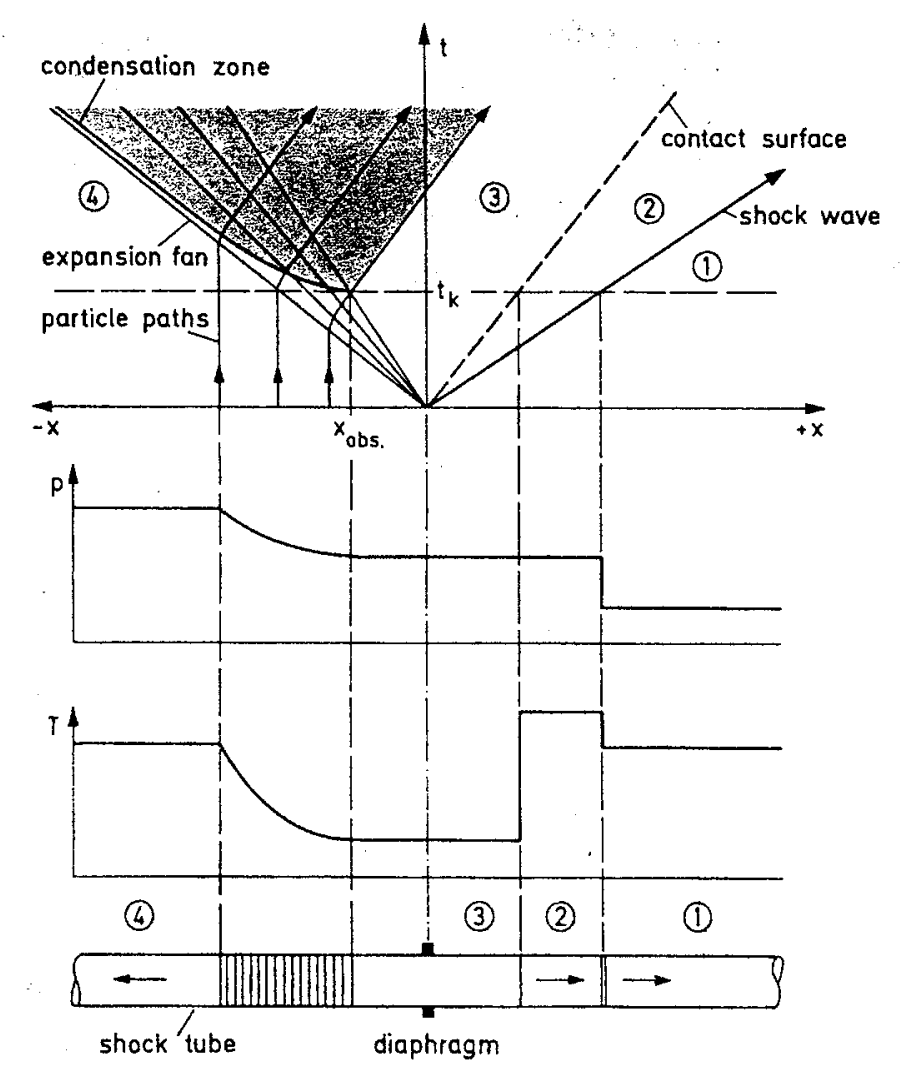

Fig. (1) Principle of shock tube operation.
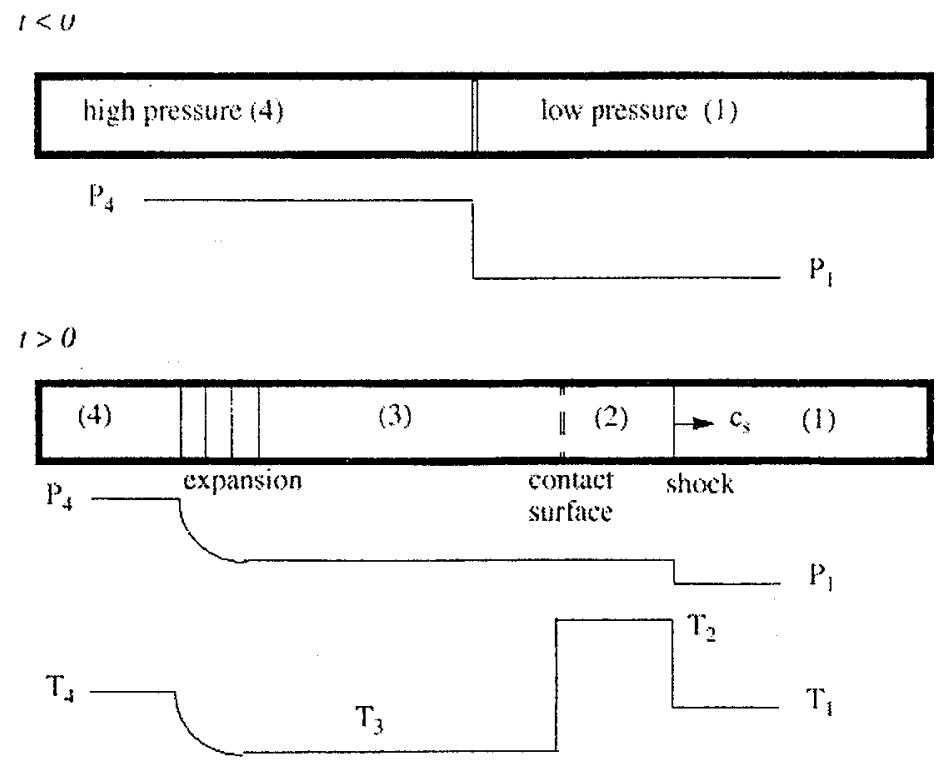

Fig. (2) Representation of the flow properties variation during the shock tube operation. 


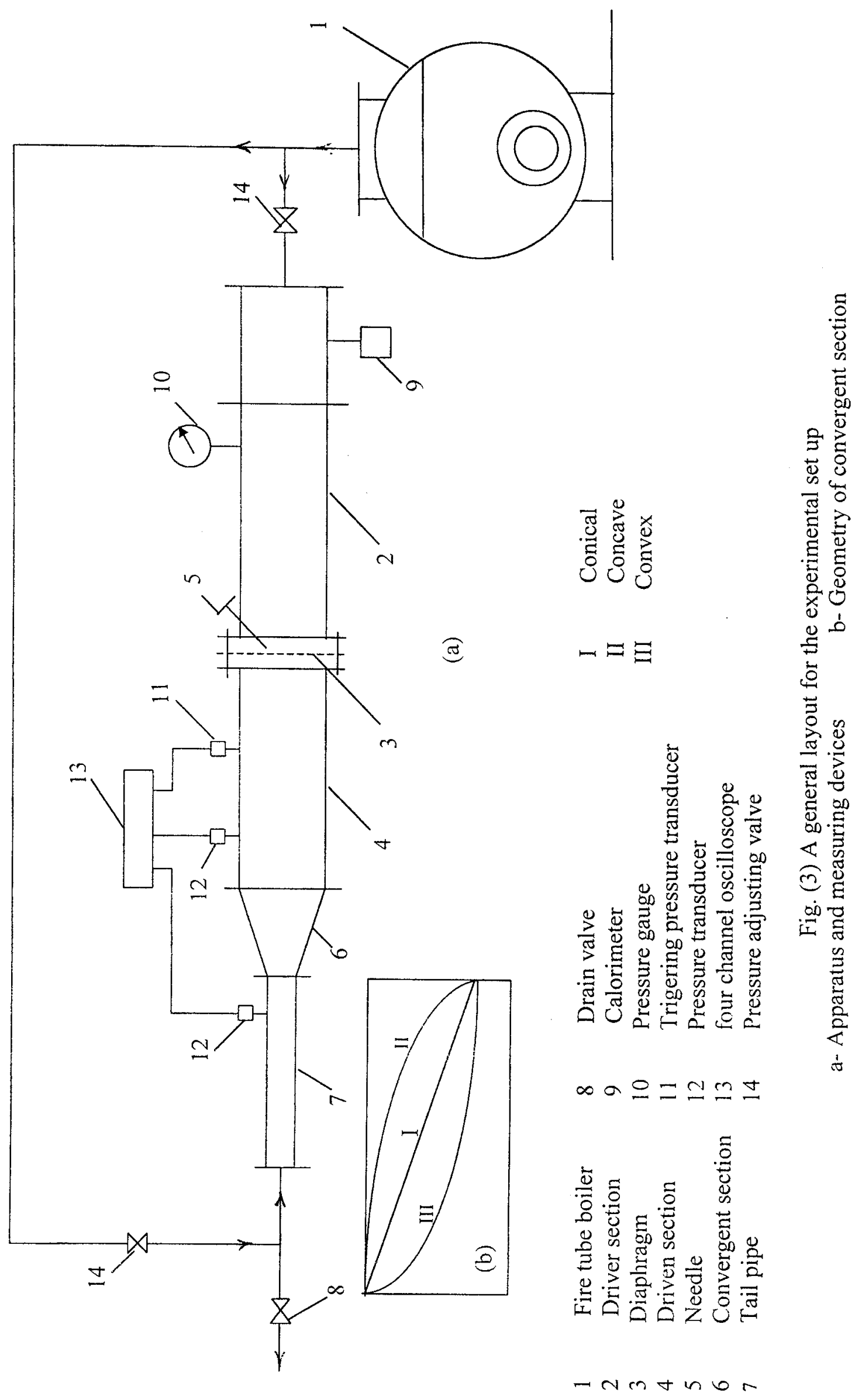




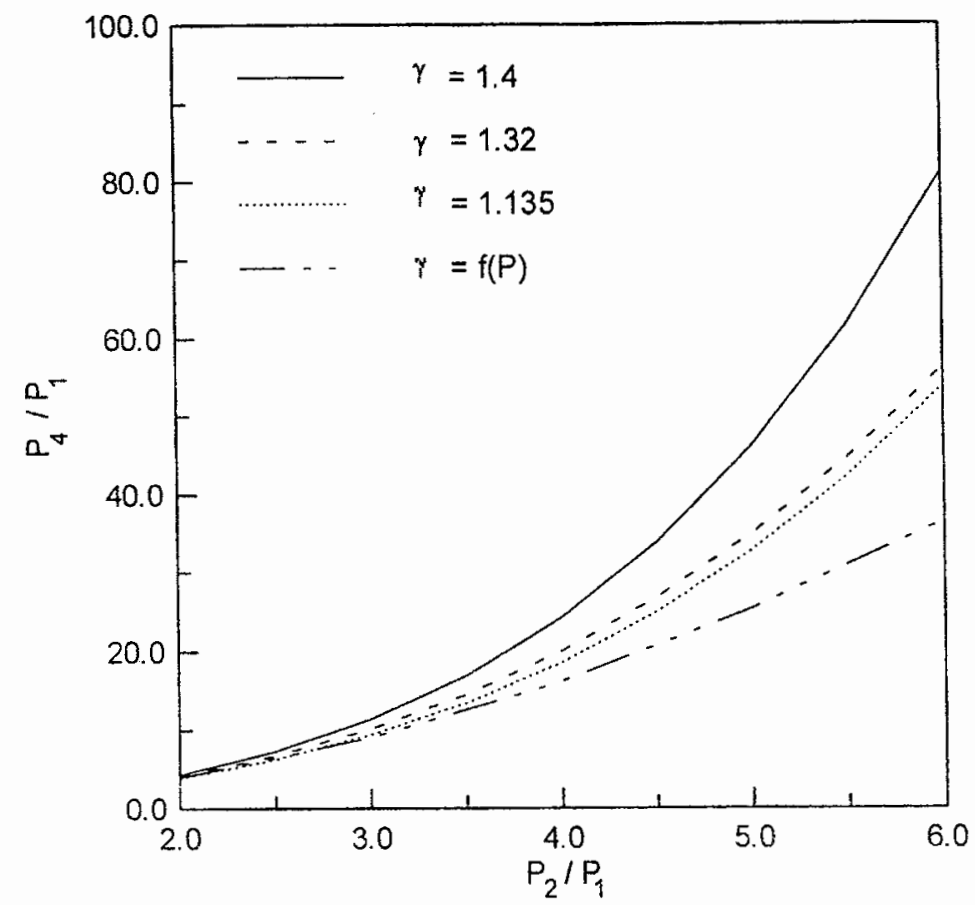

Fig. (4) Combination of a shock tube pressure ratios according to Equ. (6).

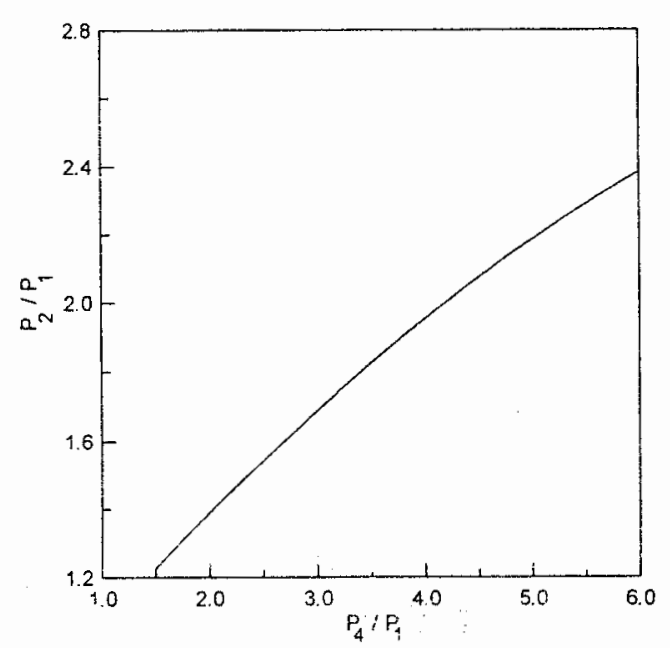

(a)

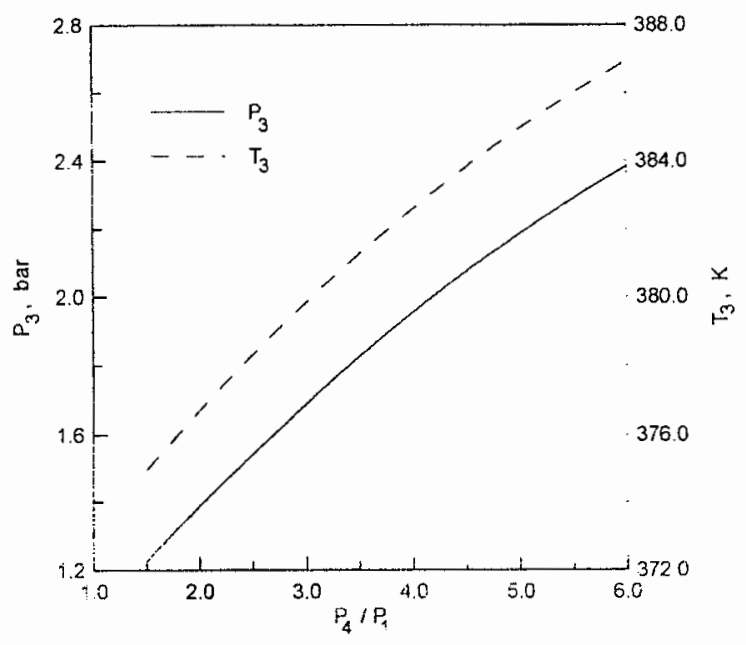

(b)

Fig. (5) Predicted characteristics of a basic shock tube filled with steam according to Equ. (7).

a- Combination of the pressure ratios

b- Dependence of $\mathrm{P}_{3}$ and $\mathrm{T}_{3}$ on $\mathrm{P}_{4} / \mathrm{P}_{1}$. 


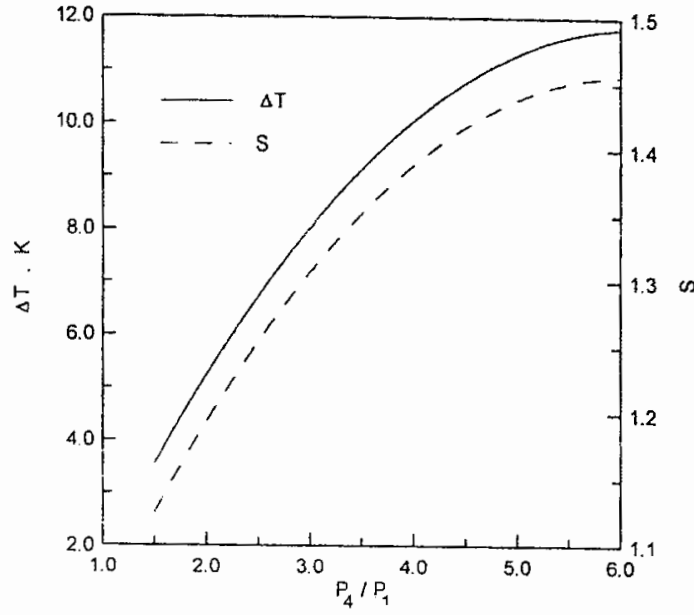

(a)

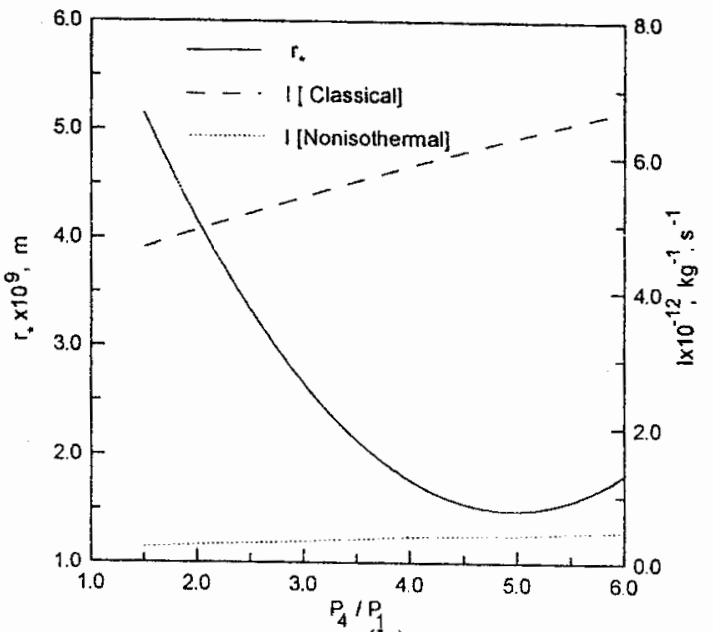

(b)

Fig. (6) Variation of steam condensation characteristics with the initial pressure ratio.

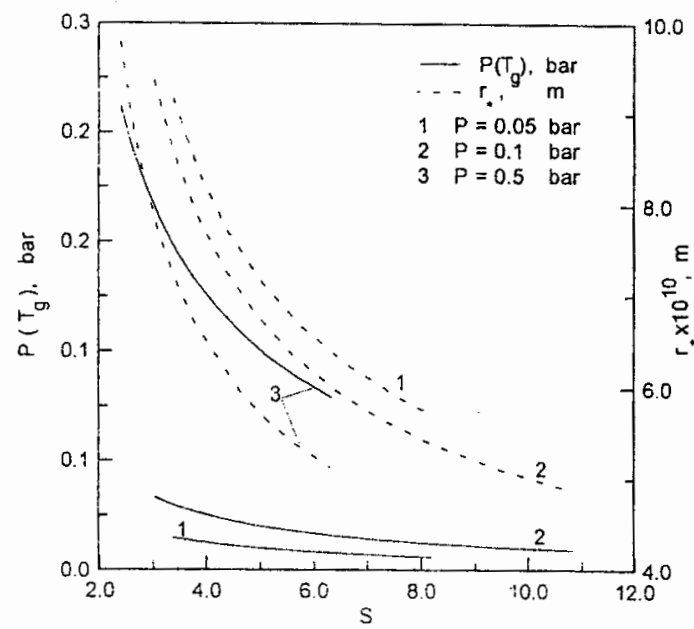

(a)

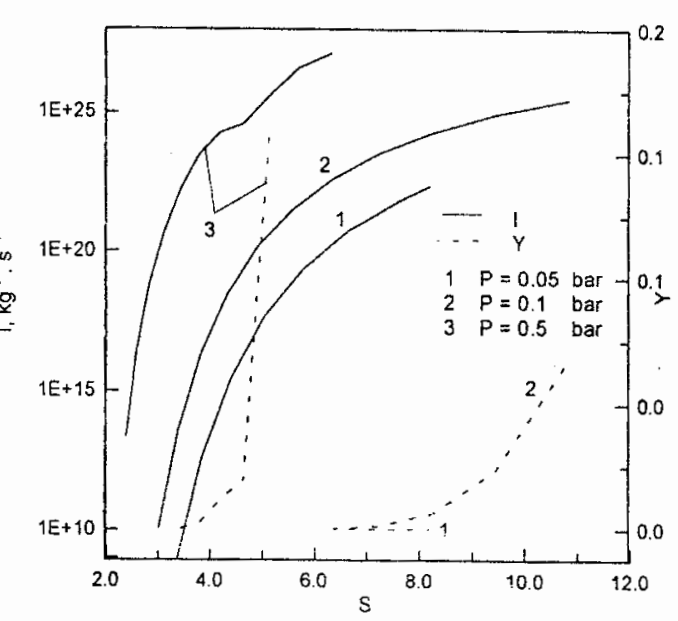

(b)

Fig. (7) Variation of the predicated condensation characteristics with different pressures of steam inside the driver section of a basic shock tube. 


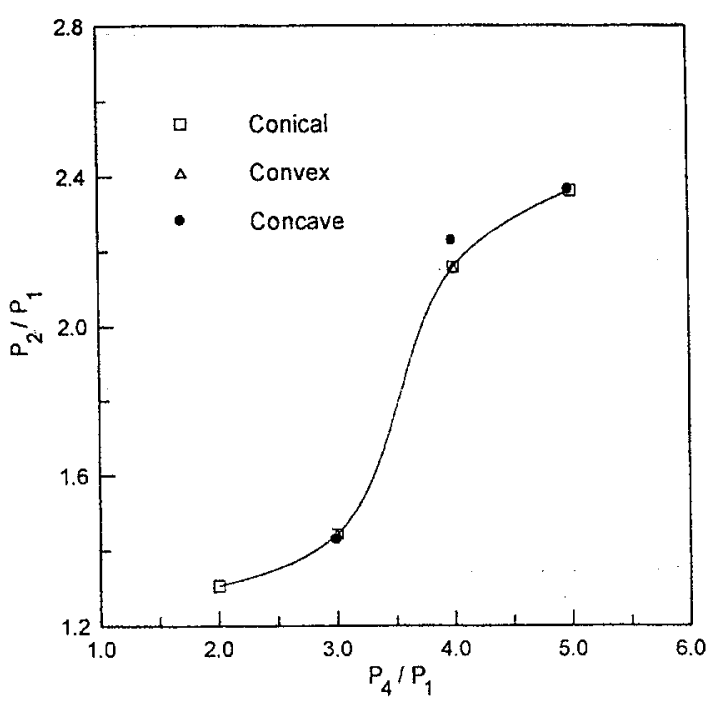

(a)

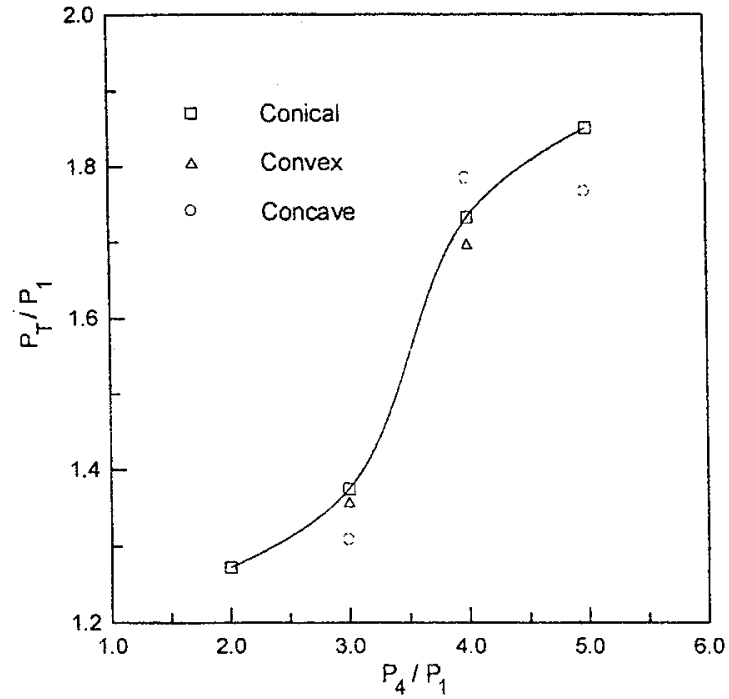

(b)

Fig. (8) Influence of both the convergent section geometry and the initial pressure ratio on the strength of :

a- the incident shock wave, b- the transmitted shock wave.

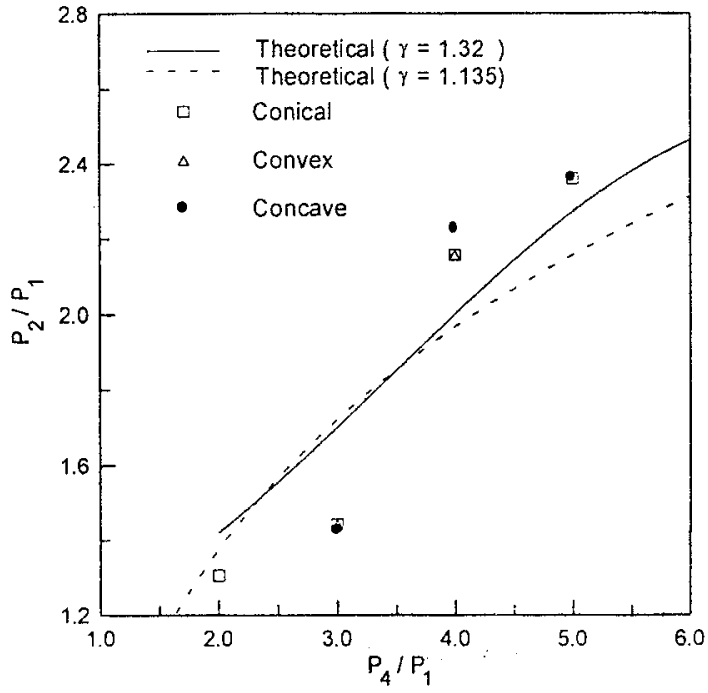

(a)

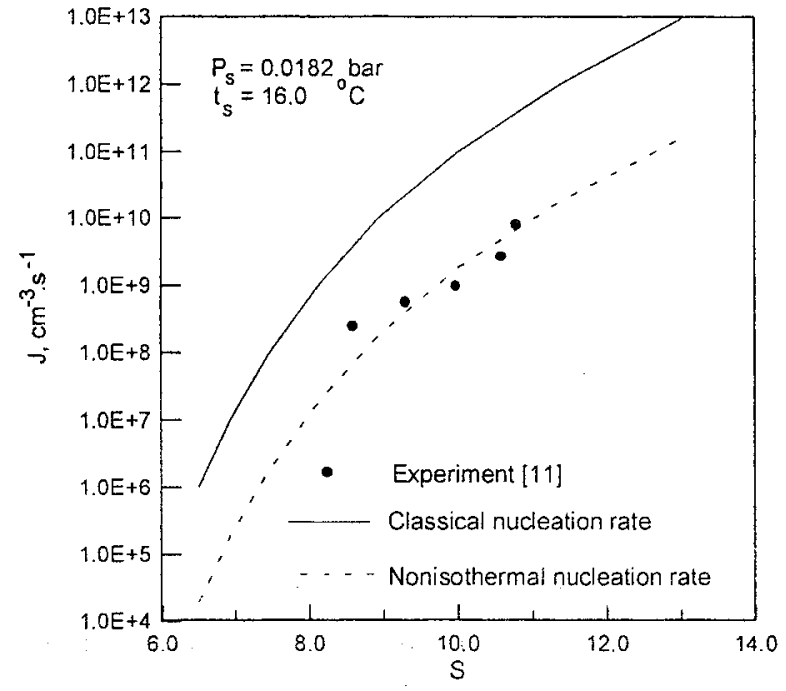

(b)

Fig. (9) A comparison between the predicted and measured values of : a- shock tube pressure ratios, b- nucleation rate as a function of supersaturation ratio. 


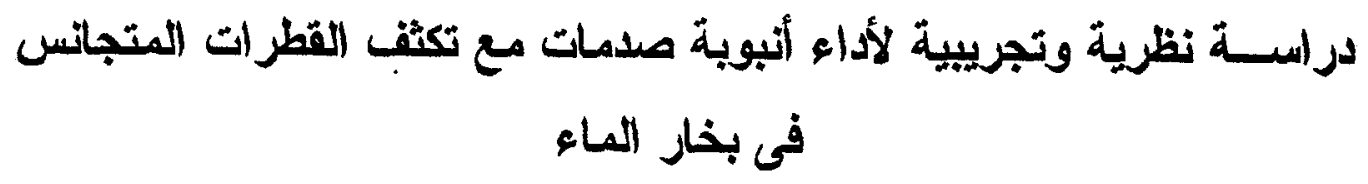

د. نبيل حنفى محمــــود

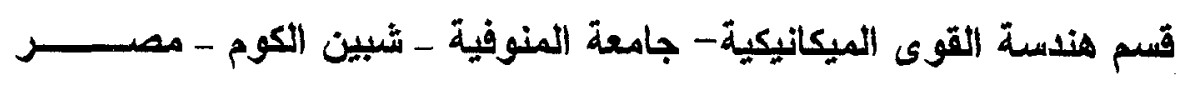

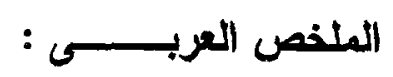

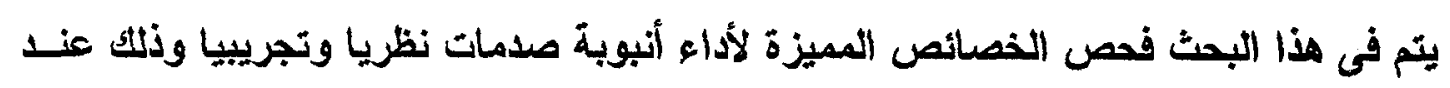

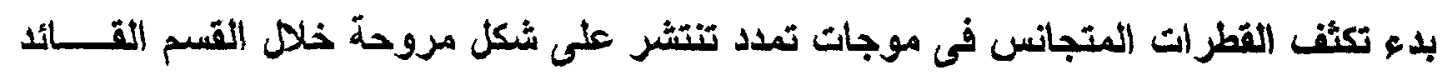

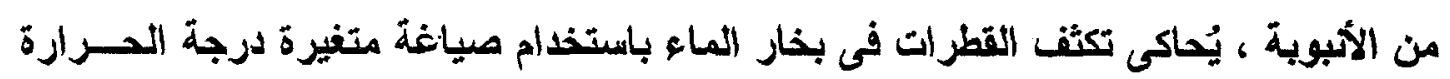

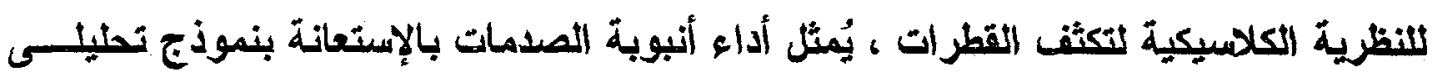

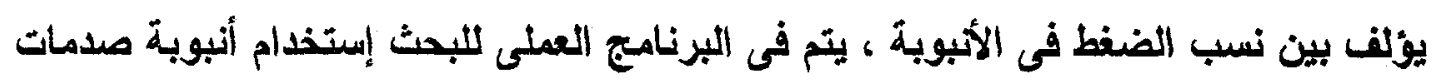

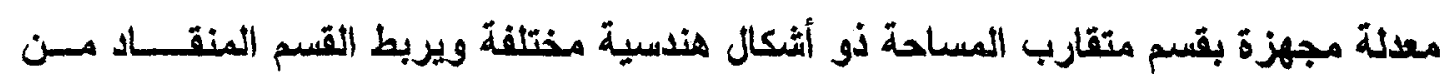

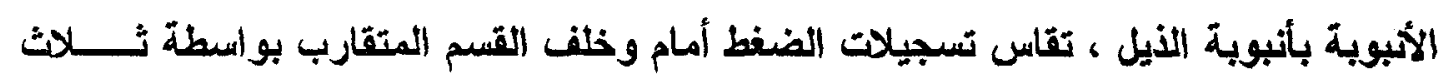

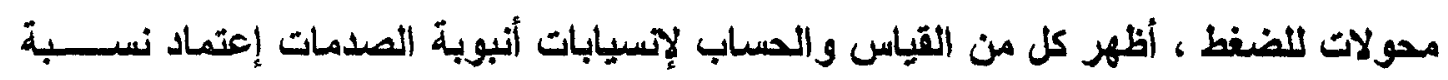

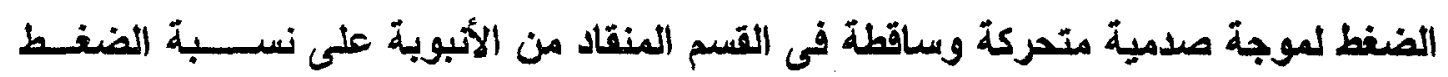

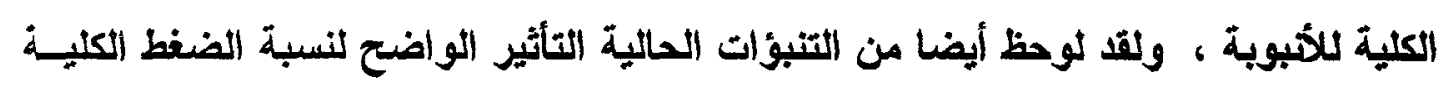

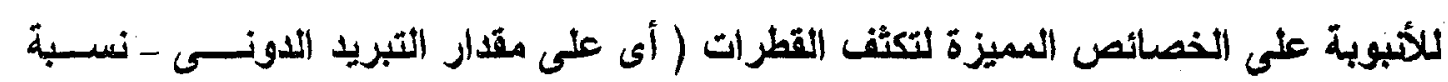

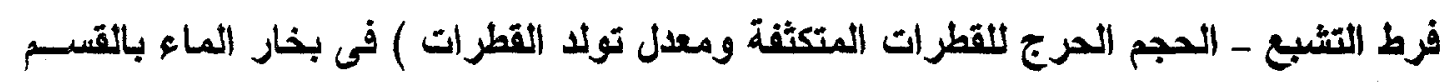

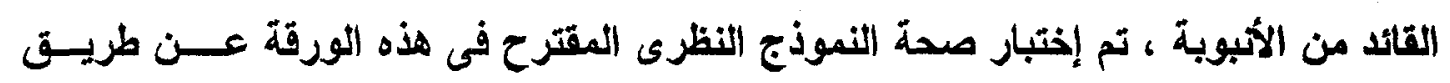

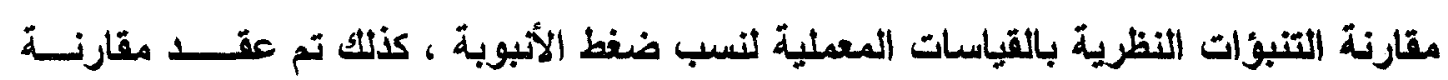

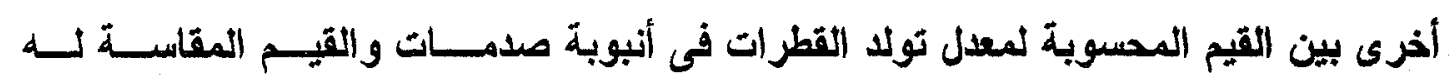
والمنشورة قبلا ، ولقد أظهرت كل من المقارنتين توافقا معقولا . 